Reprod. Nutr. Dévelop., 1988, 28 (6 B), 1763-1772

\title{
Moment de la transplantation embryonnaire et succès de la gestation chez l'humain
}

\author{
Jacqueline MANDELBAUM, Anne-Marie JUNCA, Michelle PLACHOT, \\ J. COHEN $\left({ }^{*}\right)$, J. SALAT-BAROUX $\left({ }^{* *}\right)$
}

U 173 INSERM Groupe de Recherches sur la Fécondation in vitro, Hôpital Necker-Enfants-Malades, 149, rue de Sèvres, 75743 Paris Cedex 15

(*) Service de Gynécologie-Obstétrique, CHI Jean Rostand. 141. Grande Rue, 92310 Sèvres.

$\left(^{* *}\right)$ Maternité Guy Le Lorier,

Hopital Tenon, 3, rue de la Chine, 75020 Paris.

Summary. Timing of embryo transfer and pregnancy rate in humans.

The best moment for human embryo transfer has not yet been accurately determined. The human uterus is able to receive young embryos (2, 4 cell-stage and even pronucleated eggs), insure their growth and implantation at a rate that does not exceed, however, $15 \%$ for one transferred egg.

At the present time in vitro culture to the blastocyst stage, which impairs human embryo viability, should be avoided.

Contrary to classical IVF, it becomes possible to dissociate embryo and endometrial ages when transferring frozen-thawed eggs. Our study concerns 443 spontaneous, stimulated or artificial cycles, performed in patients with normal or without endogenous ovarian function (tabl. 1). There was trend towards enhanced pregnancy rates $(17 \%)$ for synchronous as compared to one-day asynchronous transfers ( $9 \%$ ) (tabl. 2). Similar data, widely stated in mammals, emphasize the necessity of a precise chronology of embryo transfer. Embryos only survive and get implanted when placed in a receptive uterus. The onset of the refractory period appears to be closely linked to the maternal steroid environment. Consequently, no pregnancies arose from transfer of donated embryos beyond 6 days of progestative supply in women deprived of endogenous ovarian function.

In the same way, administration of progesterone $40 \mathrm{~h}$ before oocyte recovery seemed to advance the refractory phase. Indeed, a reduction in the pregnancy rate per transfer was observed in such circumstances without any obvious impairment of embryo viability (tabl. 4).

The best pregnancy rate was obtained in synchronous transfers while a one-day disynchronization reduced this rate by half.

\section{Introduction.}

L'implantation de l'œuf est le point culminant d'un dialogue où les deux protagonistes : l'œuf et l'endomètre doivent avoir acquis la maturité nécessaire à un échange fructueux. L'embryon atteint cette maturité au stade de blastocyste et l'endomètre au cours de la phase dite de «réceptivité ». Tout décalage entre le 
développement de l'embryon et celui de l'endomètre entrave le processus d'implantation. Le passage de substances diffusibles de l'un à l'autre semble être un aspect majeur de l'établissement de la grossesse chez les mammifères.

Parmi les exemples de réponse maternelle aux signaux embryonnaires citons la prolongation de la vie fonctionnelle du corps jaune et la modification localisée de perméabilité vasculaire endométriale qui précède l'implantation de plusieurs espèces. Par ailleurs, la diapause embryonnaire associée à une implantation différée chez les rongeurs semble consécutive à l'action de facteurs utérins limitant la croissance et le métabolisme du blastocyste. Qu'en est-il dans l'espèce humaine? Grâce à la technique de fécondation in vitro et transfert embryonnaire (FIVETE), il est maintenant possible de progresser plus avant dans l'étude de ces interrelations entre l'embryon préimplantatoire et l'environnement maternel.

Nous envisagerons successivement le moment de la transplantation embryonnaire au cours des cycles de FIV, des cycles de transfert d'embryons congelés et lors des dons d'ovocytes. Nous analyserons également l'effet qu'une asynchronie induite par des perturbations hormonales peut avoir sur le succès des transferts.

\section{Le moment du transfert d'embryons au cours des cycles de FIV : l'âge de l'embryon.}

L'embryon humain obtenu par fécondation externe devrait logiquement être transféré dans l'utérus au moment où il y serait théoriquement arrivé in vivo après son cheminement tubaire, soit 3 à 4 jours après la fécondation lorsqu'il est à un stade de 8 à 16 cellules (Hertig et Rock, 1949; Croxatto et al., 1972). Ce fut le cas pour les premiers transferts (Edwards et al., 1980) qui permirent l'obtention de grossesses. Puis des succès furent rapportés à partir de transferts plus précoces au stade 2 ou 4 cellules (Trounson et Wood, 1981) voire même au stade des pronuclei (Ahuja et al., 1985). Craft et al. (1982) avaient même tenté de placer dans la cavité utérine ovocytes et spermatozoïdes $2 \mathrm{~h}$ après leur mise en contact, avec il est vrai un faible taux de succès et un engouement très bref pour cette technique qui n'était pas sans rappeler les rares réussites de l'intervention d'Estes (1934) ou fécondation in utéro après inclusion de l'ovaire dans la paroi utérine. Ainsi l'utérus de la femme est capable de recevoir des zygotes très jeunes, d'assurer leur développement et leur implantation.

Marston et al. (1977) chez le singe rhésus avaient également montré que des œufs fécondés de 1 à 2 cellules transférés chirurgicalement de la trompe à l'utérus étaient capables de s'implanter. Chez la ratte la même expérience conduit à un taux réduit (30\%) mais non négligeable d'implantations (Psychoyos et Martel, 1985). Rien d'étonnant donc à ce qu'il en soit de même chez l'humain, ce qui ne prouve pas que replacer l'embryon $40 \mathrm{~h}$ environ après la fécondation, comme c'est habituellement le cas, soit le moment optimal du transfert. Le taux de grossesse par embryon transféré au cours de la FIVETE ne dépasse en effet pas $15 \%$ (Plachot et al., 1988 ; Bolton, 1988) mais divers facteurs peuvent expliquer ces résultats tels que la qualité intrinsèque de l'embryon et l'adéquation du milieu maternel au cours de cycles fortement stimulés. 
De plus, il semble que l'on ait tout intérêt à ne pas prolonger la culture de l'embryon humain in vitro. Ainsi Buster et al. (1985) montrent que $108 \mathrm{~h}$ en moyenne après l'ovulation on obtient après lavage utérin de femmes donneuses $33 \%$ de blastocystes (15/118), $17 \%$ de morulae et $50 \%$ d'embryons plus jeunes ( 2 à 16 cellules). Or Bolton (1988) en cultivant pendant $96 \mathrm{~h}$ des embryons humains obtenus par FIV et non transférés in utéro ne parvient qu'à $17 \%$ de blastocystes expansés $(55 / 317)$. Se basant sur l'hypothèse que la culture avait permis la sélection des seuls embryons viables, Bolton a alors réalisé des transferts tardifs : 1 grossesse a été obtenue sur les 8 transferts de morulae (13\%) et 2 sur les 12 transferts de blastocystes (17\%). II est difficile cependant de préciser l'origine de cette contre-performance : altération de la viabilité embryonnaire par la technique de culture ou perte d'un signal indispensable de l'embryon à la mère au cours de ces transferts tardifs ? Quoiqu'il en soit, si le moment idéal du transfert au cours de la FIVETE n'est pas encore déterminé avec certitude, le moins mauvais demeure le transfert d'un embryon de 2 à 4 cellules dans un utérus synchrone.

\section{La « fenêtre » du transfert des embryons : synchronie et asynchro- nie.}

Au cours d'un cycle classique de FIVETE, le transfert est nécessairement synchrone puisque succédant au recueil d'ovocytes préovulatoires chez la même femme. Il peut ne plus en être de même au cours des transferts d'embryons congelés. Nous avons ainsi associé à notre protocole FIVETE un programme de congélation qui permet la conservation des embryons restants après le transfert de 3 à 4 embryons frais (Mandelbaum et al., 1987a, 1988). Lorsque le cycle initial de FIV s'est soldé par un échec ou après la naissance d'un premier enfant, en cas de succès, le couple peut ainsi demander la décongélation et le transfert in utéro des embryons conservés.

De janvier 1986 à mai 1987 nous avons procédé à 443 transferts d'embryons congelés, qui après réchauffement conservaient $50 \%$ au moins de leurs blastomères intacts (tabl. 1). Le cycle de transfert était tantôt spontané avec monitorage de l'ovulation, tantôt légèrement stimulé par Clomid ou hMG en cas de dysovulation avec ou sans injection ovulante d'hCG. Une substitution par estradiol et progestérone a été proposée pour 45 transferts d'embryons congelés issus de don d'ovocytes chez des femmes dépourvues de fonction ovarienne endogène (Mandelbaum et al., 1987b). Un cycle artificiel a même été institué chez des patientes normo-ovulantes dont la fonction ovarienne avait été préalablement inhibée par les agonistes de la LHRH (52 transferts). Le taux de grossesses évolutives par transfert est identique (13 à $15 \%$ ) que le cycle ait été spontané, stimulé ou artificiel au cours des dons. Curieusement, lors des cycles artificiels chez des femmes normales on observe une réduction de ce taux (4\%) sans valeur statistiquement significative cependant sur cette courte série.

Les transferts effectués au cours des cycles spontanés ont été majoritairement synchrones ( $70 \%$ des cas), l'âge de l'embryon correspondant à l'âge de l'endomètre postovulatoire, déterminé par le dosage de la $\mathrm{LH}$ plasmatique 
TABLEAU 1

Transferts d'embryons congelés après FIV.

\begin{tabular}{lccr}
\hline \multicolumn{1}{c}{ Cycles } & Transferts & $\begin{array}{c}\text { Grossesses cliniques } \\
(\%)\end{array}$ & $\begin{array}{c}\text { Grossesses évolutives } \\
(\%)\end{array}$ \\
\hline $\begin{array}{l}\text { Cycles spontanés } \\
\text { Cycles stimulés }\end{array}$ & 262 & $40(15 \%)$ & $36(14 \%)$ \\
$\begin{array}{l}\text { Cycles artificiels } \\
\text { Cycles artificiels } \\
\text { (don d'ovocytes) }\end{array}$ & 54 & $16(19 \%)$ & $13(15 \%)$ \\
\hline
\end{tabular}

$\chi^{2}$ test: NS.

(tabl. 2). Cependant, un décalage d'1 à 2 jours a parfois été toléré. Déjà Chang, en 1950 avait montré que la survie intrautérine de l'œuf fécondé et son implantation chez la lapine nécessitait un parfait ajustement entre l'âge de l'embryon et celui de l'utérus. Depuis, les expériences de transfert d'embryons de femelles donneuses à des femelles receveuses de rat (Dickmann et Noyes, 1960), de souris (Doyle et al., 1963), de brebis (Moore et Shelton, 1964) et de vache (Rowson et al., 1972) ont montré l'importance d'une exacte synchronisation embryon-endomètre, même si les limites autorisant un transfert fructueux peuvent s'étendre à plusieurs jours chez certaines espèces comme la truie (Polge, 1982), la jument (Oguri et Tsutsumi, 1982) et le singe (Hodgen, 1983).

TABLEAU 2

Transferts d'embryons conge/és après FIV au cours de cycles spontanés.

\begin{tabular}{cccc}
\hline $\begin{array}{c}\text { Synchronie embryon/ } \\
\text { endomètre }\left({ }^{*}\right)\end{array}$ & Transferts & $\begin{array}{c}\text { Grossesses cliniques } \\
(\%)\end{array}$ & $\begin{array}{c}\text { Grossesses évolutives } \\
\text { (\%) }\end{array}$ \\
\hline 0 & 168 & $32(19 \%)$ & $29(17 \%)$ \\
-1 & 23 & $2(9 \%)$ & $2(9 \%)$ \\
+1 & 44 & $5(11 \%)$ & $4(9 \%)$ \\
+2 & 5 & 1 & 1 \\
Ovulation & 22 & 1 & $/$ \\
imprécise & & & \\
\hline
\end{tabular}

$\chi^{2}$ test: NS.

(*) Lorsque l'âge de l'embryon et celui de l'endomètre postovulatoire coïncident, le degré de synchronie a été désigné par $0 ;-1$ correspond à un endomètre plus jeune d' 1 jour que l'embryon et +1 et +2 à des endomètres en avance $d^{\prime} 1$ ou 2 joưrs par rapport à l'âge embryonnaire.

Dans notre étude de transfert des embryons congelés les résultats sont sensiblement meilleurs en terme de grossesses évolutives par transfert lorsque le transfert a été synchrone $(17 \%)$ plutôt que décalé d'1 jour $(9 \%)$, bien que les différences ne soient pas statistiquement significatives. Des données similaires 
ont été rapportées par Trounson (1986) et Quinn et Kerin (1986). On remarquera cependant que dans notre série, l'implantation reste possible malgré une asynchronie d' 1 jour. II en va de même dans l'expérimentation d'autres mammifères où le taux de grossesse ne chute dramatiquement que si l'asynchronie dépasse $24 \mathrm{~h}$ à $48 \mathrm{~h}$ suivant les espèces.

Pourquoi ce nécessaire ajustement entre l'âge de l'embryon et l'âge de l'endomètre et pourquoi un certain degré d'asynchronie est-il possible ? L'utérus semble pouvoir moduler le rythme du développement embryonnaire. Ainsi, placés dans un utérus plus âgé, les embryons ont une croissance accélérée tandis que transférés dans un utérus plus jeune ils ont une croissance ralentie (brebis, Lawson et al., 1983; Wilmut et al., 1986). Ces variations tendent à ramener l'embryon à un stade de développement compatible avec l'âge utérin. Cependant, si l'asynchronie est trop grande, les embryons présentent des anomalies, ne s'implantent pas et/ou dégénèrent. On a alors dépassé la phase de réceptivité et la survie intrautérine de l'embryon n'est plus possible. La durée de cette phase réceptive est sans doute variable suivant les espèces et conditionnée par l'environnement hormonal stéroïdien mais elle s'achève invariablement à un moment précis de la phase lutéale. C'est alors la phase réfractaire durant laquelle l'endomètre ne peut plus avoir de réaction déciduale tandis que le milieu utérin devient hostile à l'embryon non implanté. Cette hostilité pourrait résulter soit de l'absence d'un facteur essentiel à la survie embryonnaire, soit de la présence d'un facteur toxique. Ainsi $100 \%$ des blastocystes de rat cultivés en présence de perfusats d'utérus réfractaire $\left(J_{6}\right)$ dégénèrent très rapidement (Psychoyos et Casimiri, 1981) ; il en va de même chez la souris (Weitlauf, 1978). De même, les fluides utérins recueillis au cours de l'implantation différée de la souris contiennent un inhibiteur dialysable du développement embryonnaire (Aitken, 1977) qui pourrait expliquer l'entrée en diapause de l'embryon et qui disparaît d'ailleurs lors de la réactivation du blastocyste par les estrogènes. Dans l'espèce humaine, Aitken et Maathuis (1978) ne retrouvent un effet embryotoxique des lavages utérins qu'en fin de phase lutéale avec réduction significative de l'éclosion du blastocyste de souris in vitro. Au cours de la phase sécrétoire moyenne l'apparition de protéines nouvelles a été rapportée (Shirai et al., 1972; Joshi et al., 1980) mais leur signification reste cependant obscure.

\section{La cinétique de développement des embryons ou l'asynchronie embryonnaire.}

Nous venons de voir que l'environnement utérin n'est permissif à l'embryon préimplantatoire que brièvement. II exerce ainsi de fortes pressions sélectives de façon à ce que seuls les zygotes se développant de façon synchrone à l'endomètre survivent. Ceux qui croissent trop lentement par rapport à l'âge endométrial seront éliminés.

C'est peut-être un des éléments expliquant le plus fort taux d'implantation des embryons qui ont le développement le plus rapide au moment du replacement unique in utéro lors des tentatives de FIVETE chez la femme (Trounson et Mohr, 
1983; Claman et al., 1987 ; Plachot et al., 1988), d'autant que l'échange de signaux entre la mère et l'embryon opère dans les 2 directions et est la source d'interactions locales synchronisées entre blastocyste et utérus. Les embryons les plus avancés pourraient ainsi modifier l'environnement utérin qui devient hostile aux œufs plus jeunes (Pope et al., 1986). Ainsi, s'il existe une hétérogénéité de développement des embryons au cours des transferts multiples, ce sont les embryons les plus avancés qui devraient avoir les meilleures chances de s'implanter.

\section{L'asynchronie induite ou la désynchronisation pharmacologique.}

La séquence hormonale qui permet l'acquisition de la réceptivité utérine est bien connue chez le rat (Psychoyos, 1976). Une exposition à la progestérone pendant au moins $48 \mathrm{~h}$ entraîne une augmentation des récepteurs à l'estradiol du stroma endométrial. L'utérus est alors dans un état neutre mais capable de répondre à un stimulus estrogénique qui déclenche une brève phase réceptive suivie d'une phase réfractaire.

$\mathrm{Si}$ l'administration de progestérone préovulatoire désynchronise les relations ovoendométriales en avançant la séquence $P-E_{2}$, l'œuf fécondé entrera dans la cavité utérine face à un environnement hostile. Ainsi, $5 \%$ seulement des embryons de lapin de 3 jours exposés 24 heures à un utérus traité depuis l'avant veille de l'ovulation par de la progestérone à doses physiologiques s'implantent lorsqu'ils sont retransférés chez une receveuse synchrone témoin. Le traitement progestéronique préovulatoire continu a déclenché une embryotoxicité utérine (Schacht et Foote, 1978). II en est de même chez le rat où le taux d'implantation des morulae passe de $49 \%$ à 38,13 et $2 \%$ lorsque le transfert est précédé de 2 , 3,4 ou 5 jours de traitement par la progestérone (Dickmann, 1970).

Qu'en est-il dans l'espèce humaine.

Une désynchronisation pharmacologique a été réalisée involontairement dans notre équipe (Cohen, comm. personnelle, 1988). Une erreur de transmission a conduit à administrer de la progestérone micronisée par voie vaginale (Utrogestan, Besins-Iscovesco) à la dose de 200 à $300 \mathrm{mg}$ par jour à partir du jour de l'injection ovulante d'hCG chez des patientes en FIVETE. Au cours d'une période de 4 mois (tabl. 3) le nombre moyen d'ovocytes recueillis par ponction, le taux d'embryons replacés et congelés et ce faisant la qualité embryonnaire n'a en rien différé de la période précédente où la progestérone avait été administrée à partir du jour du transfert. Cependant le taux de grossesses cliniques par transfert (tabl. 4) était significativement réduit de $21 \%$ à $12 \%$ et remonta tout aussi significativement à $26 \%$ dès la correction des administrations de progestérone.

On retrouve ici des résultats analogues à ceux de l'asynchronie induite par la progestérone chez l'animal. Quelle est la part d'une altération de la réceptivité endométriale ou d'une embryotoxicité du milieu utérin dans ce phénomène ? La question reste posée. On peut rapprocher de ces observations les résultats des transferts d'embryons chez des femmes dépourvues de fonction ovarienne 
TABLEAU 3

Résultats biologiques de la FIV au cours de l'asynchronie induite.

\begin{tabular}{lccc}
\hline $\begin{array}{c}\text { Traitement } \\
\text { progestagène (") }\end{array}$ & $\begin{array}{c}\text { Nombre moyen } \\
\text { d'ovocytes/ponction }\end{array}$ & $\begin{array}{c}\text { Nombre moyen } \\
\text { d'embryons/transfert }\end{array}$ & $\begin{array}{c}\text { Embryons } \\
\text { transférés + congelés/ } \\
\text { ovocytes totaux }\end{array}$ \\
\hline A partir de l'injection d'hCG & 7,8 & 3,5 & $899 / 1790(50 \%)^{\mathrm{a}}$ \\
A partir du recueil d'ovocytes & 8,8 & 3,3 & $349 / 814 \%(43 \%)^{\mathrm{b}}$ \\
A partir du transfert & 6,9 & 3,2 & $806 / 1623(50 \%)$ \\
\hline
\end{tabular}

$\chi^{2}$ test : $a-b: p<0,001$.

(*) Utrogestan vaginal : 200 à $300 \mathrm{mg} / \mathrm{jour}$.

TABLEAU 4

Résultats des transferts d'embryons au cours de l'asynchronie induite.

\begin{tabular}{lcc}
\hline Traitement progestagène & Transferts & Grossesses cliniques (\%) \\
\hline A partir de l'injection d'hCG & 203 & $24(12 \%)^{b}$ \\
A partir du recueil d'ovocytes & 72 & $19(26 \%)^{\mathrm{c}}$ \\
A partir du transfert & 206 & $44(21 \%)^{\mathrm{a}}$ \\
\hline
\end{tabular}

$\chi^{2}$ test : $\mathrm{a}-\mathrm{b}: \mathrm{p}<0,01$,

b-c: $p<0,01$.

endogène et recevant un traitement hormonal substitutif. En effet, il est alors possible de prévoir l'environnement hormonal et histologique de l'endomètre artificiellement stimulé.

Les transferts au moment supposé le plus adéquat, soit 2 à 4 jours après l'introduction du composé progestatif (Lutjen et al., 1987 ; Devroey et al., 1987 ; Mandelbaum et al., 1987b) ont conduit à des grossesses. Les transferts plus tardifs, au $6^{\mathrm{e}}$ jour ou plus de l'imprégnation progestéronique, n'ont pas permis d'obtenir d'implantation (Navot et al., 1986 ; Rosenwaks, 1987).

$27^{e}$ Réunion de la Société française pour l'Etude de la Fertilité. Paris, 29, 30 sept., $1^{\text {er }}$ oct. 1988.

\section{References}

AHUJA K. K., SMITH W., TUCKER M., CRAFT I., 1985. Successful pregnancies from the transfer of pronucleate embryos in an outpatient in vitro fertilization program. Fertil. Steril., 44, 181 - 184.

AITKEN R. J., 1977. The culture of mouse blastocysts in the presence of uterine flushings collected during normal pregnancies, delayed implantation and proestrus. J. Emb. exp. Morph., 41. 295-300.

AITKEN R. J., MAATHUIS J. B., 1978. Effects of human uterine flushings collected at various states of the menstrual cycle on mouse blastocysts in vitro. J. Reprod. Fertil., 53, 137. 
BOLTON V. N., 1988. Fécondation in vitro et transplantation d'embryons au stade de blastocyste tardif. Contr. Fert. Sex, 16, 375-377.

BUSTER J. E., BUSTILLO M., BODI I. A., COHEN S.W., HAMILTON M., SIMON J.A., THORNEYCROFT I. H., MARSHALL J. R., 1985. Biologic and morphologic development of donated human ova recovered by nonsurgical uterine lavage. Am. J. Obstet. Gynecol., 153, $211-217$.

CHANG M. C., 1950. Development and fate of transferred rabbit ova or blastocyst in relation to the ovulation time of recipients. J. exp. Zool., 114, 197-224.

CLAMAN P., ARMANT D. R., SEIBEL M. M., WANG T., OSKOWITZ S. P., TAYMOR M. L., 1987. The impact of embryo quality and quantity on implantation and the establishment of viable pregnancies. J. IVF, 4, 218-222.

CRAFT I., Mc LEOD F., GREEN S., DJAHANBAKHCH O., BERNARD A., TWIGG H., SMITH W., LINDSAY K., EDMONDS K., 1982. Human pregnancy following oocyte and sperm transfer to the uterus. Lancet, vol. $1031-1033$.

CROXATTO H. B., DIAZ S., FUENTALBA B., CROXATTO H.D., CARILlO D., FABRES C., 1972. Studies on the duration of egg transport in the human oviduct. I. The time interval between ovulation and egg recovery from the uterus in normal women. Fertil. Steril., 23. 447-458.

DEVROEY P., BRAECKMANS M., CAMUS M., KHAN I., SMITZ C., STAESSENS E., VAN DEN ABBEEL E., VAN WAESBERGHE L., WISANTO A., VAN STEIRTEGHEM A. C., 1987 . Pregnancies after replacement of fresh and frozen-thawed embryos in a donation program, 133-137. In FEICHTINGER W., KEMETER P. Future aspects in human in vitro fertilization. Springer Verlag, Berlin.

DICKMANN Z., 1970. Effects of progesterone on the development of the rat morula. Fertil. Steril., 21, 541-548.

DICKMANN Z., NOYES R. W., 1960. The fate of ova transferred into the uterus of the rat. J. Reprod. Fert., 1, 197-212.

DOYLE L. L., GATES A. H., NOYES R. W., 1963. Asynchronous transfer of mouse ova. Fert. Steril., 14, 215-225.

EDWARDS R. G., STEPTOE P. C., FOWLER R. E., BAILLIE J., 1980. Observations on preovulatory human follicles and their aspirates. Br. J. Obstet. Gynaecol., 87, 769-779.

ESTES W. L., HEITMEYER P. L., 1934. Pregnancy following ovarian implantation. Am. J. Surg., 24, 563-580.

HERTIG A. T., ROCK J., 1949. A series of potentially abortive ova recovered from fertile women prior to the first missed menstrual period. Am. J. Obstet. Gynecol., 58, 968.

HODGEN G. D., 1983 . Surrogate embryo transfer combined with estrogen-progesterone therapy in monkeys. J. am. med. Assoc., 250, 2167-2171.

JOSHI S. G., EBERT K. M., SWARTZ D. P., 1980. Detection and synthesis of a progestagen dependent protein in human endometrium. J. Reprod. Fert., 59, 273-285.

LAWSON R. A. S., PARR R. A., CAHILL L. P., 1983. Evidence for maternal control of blastocyst growth after asynchronous transfer of embryos to the uterus of the ewe. J. Reprod. Fert., 67, 447-483.

LUTJEN P. J., HEALY D. L., CHAN C., FINDLAY J. K., KOLA I., TROUNSON A. O., 1987. Oocyte and embryo donation in women with absent ovarian function, 125-132. In FEICHTINGER W., KEMETER P., Future aspects in human in vitro fertilization. Springer Verlag, Berlin.

MANDELBAUM J., JUNCA A. M., PLACHOT M., ALNOT M. O., ALVAREZ S., DEBACHE C., SALAT-BAROUX J., COHEN J., 1987a. Human embryo cryopreservation, extrinsic and intrinsic parameters of success. Human Reprod., 2, 709-715.

MANDELBAUM J., JUNCA A. M., PLACHOT M., ALNOT M. O., CORNET D., SALAT-BAROUX J., DEBACHE C., COHEN J., 1987b. Un nouveau développement de la fécondation in vitro : le don d'ovocytes. Contr. Fert. Sex., 15, 1141-1146.

MANDELBAUM J., JUNCA A. M., PLACHOT M., ALNOT M. O., SALAT-BARDUX J., ALVAREZ S., TIBI C., COHEN J., DEBACHE C., TESQUIER L., 1988. Cryopreservation of human embryos and oocytes. Human Reprod., 3, 117-119.

MARSTON J. M., PENN R., SIVELLE P. C., 1977. Successful auto-transfer of tubal eggs in the rhesus monkey (Macaca mulatta). J. Reprod. Fertil., 49, 175-176. 
MOORE N. W., SHELTON J. N., 1964. Egg transfer in sheep. Effect of degree of synchronization between donor and recipient, age of egg and site of transfer on the survival of transferred eggs. J. Reprod. Fert., 7, 145-152.

NAVOT D., LAUFER N., KOPOLOVIC J., RABINOWITZ R., BIRKENFELD A., LEWIN A., GRANAT M., MARGALIOTH E. J., SHENKER J.G., 1986. Artificially induced endometrial cycles and establishement of pregnancies in the absence of ovaries. New Eng/. J. Med., 314, 806-811.

OGURI N., TSUTSUMI Y., 1982. Non surgical transfer of equine embryos, 287-295. In E.S.E HAFEZ, K. SEMM, in vitro fertilization and embryo transfer. MTP Press Ltd Lancaster.

PLACHOT M., JUNCA A. M., MANDELBAUM J., COHEN J., SALAT-BAROUX J., 1988. Les échecs du développement embryonnaire. Reprod. Nutr. Dévelop., 28, (6B), 1781-1790.

POLGE C., 1982. Embryo transplantation in the pig. In Embryo transfer in mammals. Eds Merieux C H. et Bonneau M., pp. 45-52.

POPE W. F., LAWYER M. S., NARA B. S., FIRST N. L., 1986. Effects of asynchronous superinduction on embryo survival and range of blastocysts development in swine. Biol Reprod., 35. 133-137.

PSYCHOYOS A., 1976. Hormonal control of uterine receptivity for nidation. J. Reprod. Fertil., Suppl., 25, 17-18.

PSYCHOYOS A., CASIMIRI V., 1981. Uterine blastotoxic factors, 327-334. In S. R. GLASSER, D. W. BULLOCK, Cellular and molecular aspects of implantation. Plenum Publ.

PSYCHOYOS A., MARTEL D., 1985. Problems related to the implantation of the human egg after in vitro fertilization. Acta Europ. Fert., 16, 107-109.

QUINN P., KERIN J. F. P., 1986. Experience with the cryopreservation of human embryos using the mouse as a model to establish successful techniques. J. IVF. 3, 40-45.

ROZENWAKS Z., 1987. Donor eggs : their application in modern reproductive technologies. Fertil. Steril. 47, 895-909.

ROWSON L. E. A., ROWSON R. A. S., MOOR R. M., BAKER A. A., 1972 . Egg transfer in cow ; synchronization requirements. J. Reprod. Fertil., 28, 427.

SCHACHT C. J., FOOTE R. H., 1978. Progesterone induced asynchrony and embryo mortality in rabbits. Biol. Reprod., 19, 534-539.

SHIRAI E., IIZUKA R., NOTAKE Y., 1972. Analysis of human uterine fluid protein. Fertil. Steril., 23, 522-528

TROUNSON A., 1986. Preservation of human eggs and embryos. Fertil. Steril., 46, 1-12.

TROUNSON A., MOHR L., 1983. Human pregnancy following cryopreservation, thawing and transfer of an eight-cell embryo. Nature, 305, 707-709.

TROUNSON A., WOOD C., 1981. Extracorporeal fertilization and embryo transfer. Clinics Obst. Gynaecol., 83, $681-713$.

WEITLAUF H. M., 1978. Factors in mouse uterine fluid that inhibit the incorporation of ${ }^{3} \mathrm{H}$-uridine by blastocysts in vitro. J. Reprod. Fertil., 52, $321-325$.

WILMUT I., SALES D. I., ASHWORTH C. J., 1986. Maternal and embryonic factors associated with prenatal loss in mammals. J. Reprod. Fert., 76, $851-864$. 